OPEN ACCESS

Citation: Yuri A. Kirillov, Maria A. Kozlova, Lyudmila A. Makartseva, Igor A. Chernov, Evgeniya V. Shtemplevskaya, DavidA. Areshidze (2021) Influence of chronic alcoholic intoxication and lighting regime on karyometric and ploidometric parameters of hepatocytes of rats. Caryologia 74(3): 45-51. doi: 10.36253/caryologia-1122

Received: October 26, 2020

Accepted: June 01, 2021

Published: December 21, 2021

Copyright: (c) 2021 Yuri A. Kirillov, Maria A. Kozlova, Lyudmila A. Makartseva, Igor A. Chernov, Evgeniya V. Shtemplevskaya, David A. Areshidze. This is an open access, peer-reviewed article published by Firenze University Press (http://www.fupress.com/caryologia) and distributed under the terms of the Creative Commons Attribution License, which permits unrestricted use, distribution, and reproduction in any medium, provided the original author and source are credited.

Data Availability Statement: All relevant data are within the paper and its Supporting Information files.

Competing Interests: The Author(s) declare(s) no conflict of interest.

\section{Influence of chronic alcoholic intoxication and lighting regime on karyometric and ploidometric parameters of hepatocytes of rats}

\author{
Yuri A. Kirillov ${ }^{1}$, Maria A. Kozlova ${ }^{1}$, Lyudmila A. Makartseva ${ }^{1}$, Igor \\ A. Chernov ${ }^{2}$, Evgeniya V. Shtemplevskaya ${ }^{1}$, David A. Areshidze ${ }^{1, *}$ \\ ${ }^{1}$ Federal State Budgetary Scientific Institution «Research Institute of Human Morphol- \\ ogy» \\ ${ }^{2}$ FSBEI HE Tyumen State Medical University of the Ministry of Health of Russia \\ *Corresponding author. E-mail: labcelpat@mail.ru
}

\begin{abstract}
The features of the diurnal dynamics of the area of rat hepatocyte nuclei and their ploidy were studied under conditions of a standart (fixed) light regime and constant illumination, as well as under chronic exposure to alcohol in the mentioned light regimes. It has been shown that exposure to alcohol and exposure to constant illumination separately lead to a change in the amplitude-phase characteristics of the circadian rhythm of the nucleus area, while the combined effect of these factors leads to a complete destruction of the rhythm, which indicates a violation of adaptation processes. An increase in the average ploidy of hepatocyte nuclei in chronic alcohol intoxication is also shown, while in animals kept under constant illumination without drinking alcohol, the values of this parameter decrease, which indicates a successful course of the adaptation process. The conducted research indicates that the results of karyometric and ploidometric analysis characterize the degree of influence of alcohol intoxication and changes in the light regime on the liver of rats, reflecting the rate of efficiency of adaptation to these factors.
\end{abstract}

Key words: caryometry, ploidy, hepatocyte, circadian, morphometry.

\section{INTRODUCTION}

The liver is the main organ of metabolism of various exogenous and endogenous chemical compounds, while the main functionally active liver cells (hepatocytes) are among the first to be exposed to these factors. Damage and death of these cells renders to the disability of the liver to perform its functions (Aizava et al., 2020).

One of the mechanisms for maintaining the structural and functional integrity of the liver is cellular regeneration, which occurs due to mitotic and amitotic division of hepatocytes (Gilgenkrantz et al., 2018; Clemens et al., 2019).

Mass death of hepatocytes by necrosis and/or apoptosis activates the processes that trigger the entry of "resting" hepatocytes into the cell-division cycle to restore the original cell mass and maintain the cellular homeostasis 
of the organ. The liver always has a reserve of hepatocytes with polyploid nuclei, constantly ready to divide. Thus, an increase in the level of ploidy of hepatocytes is one of the main compensatory-adaptive reactions of the liver, ensuring the preservation of function of the organ. It is known that in mammals in the prenatal and at the early stages of postnatal ontogenesis, diploid hepatocytes prevail, then their polyploidization occurs, and the proportion of polyploid hepatocytes can reach $80 \%$ of the total number of cells. In addition, it was found that ploidy of hepatocytes increases with aging, after hepatectomy, under the influence of a number of unfavorable factors, but at the same time ploidy decreases, for example, in hepatocellular carcinomas (Duncan, 2013; Zhang et al., 2019; Donne et al., 2020).

Another, very important and informative approach to determining the functional state of the liver, as well as diagnosing various kinds of diseases of this organ is karyometry. Karyometric analysis is used to assess the intensity of dystrophic, inflammatory, reparative processes in chronic viral hepatitis, liver fibrosis, hepatocellular carcinoma, etc (El-Sokkary et al., 2005; Esperandim et al., 2010; Makovsky P et al., 2018).

Thus, the approaches of the study of the liver in different morphofunctional states can be associated with the karyometric and ploidometric assessment of hepatocytes. The data obtained using these methods will make it possible to assess the morphofunctional state of the liver more accurately and, in accordance with this, to solve the problems of prognosis.

Rhythmicity of functioning is peculiar for living systems at every level of organization. Biosystems have rhythms with different periodicity, however, diurnal, or circadian rhythms (CR) are the most significant for mammals (Gillette, 2013; McKenna et al., 2018).

Circadian system of mammals includes central circadian rhythm generators (suprachiasmatic nuclei of the hypothalamus (SCN), pineal gland), which are connected with peripheral pacemakers - morphological structures in organs and tissues. It is endogenous and is determined genetically (genes Per1, Per2, Cry, etc.), however, it has significant plasticity and can be modulated by the action of external zeitgebers (time givers), the most important of which is light (Tahara et al., 2017).

Separate biorhythms of physiological processes in various systems form a strongly coordinated ensemble, the chronostructure of the organism. The presence of a rhythmic structure of biological processes ensures the necessary order of their course, coherence, maintenance of the functioning of systems of organism at an optimal level (Roennenberg et al., 2016).
Exposure to endogenous or exogenous desynchronizing factors leads to disorganization of circadian rhythmicity (Roennenberg et al., 2017). In the case of prolonged or regular exposure to desynchronizing factors, for example, constant lighting at night, desynchronosis develops, which is a pathological condition characterized by a mismatch of rhythms in phase, the loss of their mutual synchronization or their destruction (Beauvalet et al., 2017; Walker et al., 2020).

One of the organs, the normal rhythm of the functioning of which is very important for maintaining homeostasis, is the liver (Trefts et al., 2017). In the regulation and realization of plastic and energy metabolism, the coordination of rhythmic processes in the liver with the rhythms of processes in other organs and systems of the organism plays a fundamental role. Moreover, most of these processes demonstrate the daily rhythm (Tahara et al., 2016).

In most cases, the rhythm of metabolic processes arises and is maintained due to dynamic interactions between the molecular clock of the organism and external zeitgebers, such as, for example, light (main CR synchronizer) and nutrition (secondary synchronizer) (Stubblefield et al., 2016; Ding et al., 2018).

The disruption of circadian rhythmicity in the form of a shift in biorhythms or desynchronosis in the liver entails the development of pathological conditions and diseases such as cholestasis, fatty hepatosis, impaired biotransformation of toxic and medicinal substances, hepatitis, cirrhosis and liver tumors (Tong et al., 2013; DeBruyne et al., 2014). An indicator of functional changes in hepatocytes is the modification of their morphological structure, which has a wide range of variations, from subtle ultrastructural transformations to cell death. (Li et al., 2020). The linear dimensions of hepatocytes and their nuclei, nuclear-cytoplasmic ratio, and ploidy of hepatocytes are the significant parameters for assessing the morphofunctional state of the liver (Junatas et al., 2016).

The significant reason of desorganization of biorhythms in the modern world is the disturbance of natural light regime, known as light pollution. Due to a number of social reasons (prolonged interaction with digital technique, overtime and shift work, transmeridian flights, etc.), a person is currently exposed to abundant exposure to artificial lighting in the dark, which leads to a shift in the circadian rhythms of the organism, or to the development of desynchronosis (Lunn et al., 2017).

Another factor that influences CR is alcohol consumption. In a study of the effect of alcohol on rhythms in mammals, two areas of interest are distinguished. The first one considers the chrono-effecter action of alcohol, i.e. how the effects of alcohol change depending on the 
time of day in which it was consumed. The second area of interest is chronergic, with wider approach, exploring mainly the effect of alcohol on biorhythms of other parameters of organism (Wasielewski et al., 2001). Alcohol abuse and alcoholic disease are associated with widespread disturbances in CR (Rosenwasser, 2015; Davis et al., 2018). It is shown that disturbances in circadian homeostasis make liver and intestines more susceptible to alcohol toxicity. Studies in human alcoholics have shown altered expression of circadian genes. Anyway, alcohol has a significant chronotoxic effect, which causes desynchronosis (Huang et al., 2010; Filiano et al., 2013; Martínez-Salvador J. et al., 2018.)

We considered it important to study the daily dynamics of the area of hepatocyte nuclei and their ploidy under normal light conditions and under constant illumination, as well as in combination of these conditions with experimental chronic alcohol intoxication.

\section{MATHERIALS AND METHODS}

\section{Animals}

The study was conducted on 160 male Wistar rats at age of 6 months, weighing $300 \pm 20 \mathrm{~g}$. Animals were taken from the Stolbovaya nursery (the "Stolbovaya" affiliate of the FSBIS "Scientific Center for Biomedical Technologies of the Federal Medical and Biological Agency).

\section{Design of experiment}

Rats were divided into 4 equal groups. The experiment lasted 3 weeks for every group.

1st group (control): animals of the first group served as control. The individuals were housed in plastic cages with free access to water under the conditions of a fixed light regime "light-dark" (10:14 hours).

2nd group: animals of the second group were kept under the same conditions, but instead of water, a $15 \%$ ethanol ad libitum solution was offered daily as a drink.

3rd group: animals of the third group were kept under the same conditions as the animals of the first group, except for the light regime, which represented constant lighting ("light-light").

4th group: animals of the fourth group were kept under conditions of constant lighting and got 15\% ethanol ad libitum solution as a drink instead of water.

The criterion for the selection of rats in the 2 and 4 group, along with the absence of visible deviations in the state and behavior, was the initial preference for a $15 \%$ solution of ethyl alcohol to a tap water. For this, a pre- liminary experiment was carried out for 3 days in individual cages with free access to both liquids.

Euthanasia was carried out three weeks after the start of the experiment in a carbon dioxide chamber equipped with a device for the upper gas supply $(100 \%$ $\mathrm{CO}_{2}$ ) at 9.00, 15.00, 21.00 and 3.00. The chamber volume was filled with gas at a rate of $20 \%$ per minute to avoid dyspnea and pain in animals. After sacrifice, the liver was removed for morphological examination. All animal experiments were performed in according to the compliance with EC Directive 86/609/EEC and with the Russian law regulating experiments on animals.

The liver was fixed in $10 \%$ neutral buffered formalin with further passage through alcohols of increasing concentration $\left(50^{\circ}, 60^{\circ}, 70^{\circ}, 80^{\circ}\right.$, and $\left.96^{\circ}\right)$ and xylol, followed by pouring into Histomix histological medium (BioVitrum, Russia). When conducting studies of organs embedded in paraffin, serial sections with a thickness of 5-6 $\mu \mathrm{m}$ were prepared. Histological sections were made on the rotor microtome MPS-2 (USSR). Hematoxylineosin staining was carried out according to the standard technique. Stained sections were put in a BioMount mounting medium (BioVitrum, Russia).

Microscopy of histological preparations was performed using a Nikon Eclipse 80I digital microscope with use of a Nikon DI-FI digital camera (Japan). For microscopy, eyepieces $\times 10, \times 15$, lenses $\times 4, \times 10, \times 20, \times 40, \times 100$ were used. From each studied preparation, 10 digital images of randomly selected visual fields were taken at a magnification of $\times 400, \times 1000$, with the use of which karyometry were subsequently carried out, the daily dynamics of the nucleus was determined, estimated by their area. In morphometric studies, the ImageJ program (USA) with the appropriate plug-ins was used to determine the crosssectional area of hepatocyte nuclei (Broeke et al., 2015). The measurements were carried out in micrometers after preliminary geometric calibration on an object-micrometer scale digitized with the same magnification.

For ploidometry, paraffin sections were stained with methylene-green - pyronin G, with following processing of sections with RNA-ase. The hepatocyte ploidy was calculated in units of ploidy relative to the optical density of the staining results of diploid nuclei of small lymphocytes.

Micromorphometry of only mononuclear interphase hepatocytes without signs of pathological changes was carried out.

\section{Methods of statistical processing}

The obtained data were analyzed using the GraphPad Prism 6.0 program by calculating average values, 
standard deviation, and arithmetic mean error. The numerical rows characterizing the diurnal fluctuations of the studied physiological rhythms of animals were subjected to mathematical processing, on the basis of which group chronograms were drawn. We studied the form of chronograms and calculated daily average values. Statistical differences in studied parameters were determined using t-student test. A $\mathrm{p}$ value $<0.05$ was considered statistically significant.

For the statistical estimation of the amplitude and acrophase of CRs, cosinor analysis was performed, which is an international, recognized method for the unified study of biological rhythms using the CosinorEllipse2006-1.1 program. The presence of a reliable circadian rhythm was determined, as well as its acrophase and amplitude. Acrophase is a measure of the peak time of the total rhythmic variability over a 24 -hour period. The amplitude corresponds to half the total rhythmic variability in the cycle. Acrophase is expressed in hours; amplitude values are expressed in the same units as the studied variables (Cornelissen, 2014).

\section{RESULTS}

Considering the results of karyometry, we found that the cross-sectional area of hepatocyte nuclei of rats of the first three groups, which amounted to $41.79 \pm 8.13$ $\mu \mathrm{m}^{2}, 42.65 \pm 4.80 \mu \mathrm{m}^{2}$, and $42.72 \pm 5.63 \mu \mathrm{m}^{2}$, respectively, did not differ significantly from each other, but the significant decrease in this parameter up to $35.50 \pm 3.01 \mu \mathrm{m}^{2}$ in hepatocytes of animals of the fourth group was found.

The daily rhythm of the cross-sectional area of the hepatocyte nuclei of rats of 2-4 groups significantly differed from the control (Fig. 1). In particular, the maximum of area of nuclei in control group is noted at 15:00 with acute decrease to minimum at evening and nighttime $-21: 00$ and 3:00. In the second group the rhythm is less pronounced, a maximum at 15:00 is noted. In the third group, the maximum values are noted at 9:00 with a gradual decrease to a minimum at 3:00. In the fourth group, daily fluctuations in the area of hepatocyte nuclei are unreliable.

The results of the cosinor analysis of diurnal changes in the area of the nucleus indicate the presence of a reliable CR of this process in the first three groups and its destruction in the fourth group. Therewith, acrophases of rhythms in groups 1 and 3 are noted in the daytime - at $12^{21}$ and $11^{36}$, with an amplitude of 10.03 $\mu \mathrm{m}^{2}$ and $4.60 \mu \mathrm{m}^{2}$, respectively, and the acrophase of the rhythm in the second group shifts by $18^{02}$ with an amplitude of $3.37 \mu \mathrm{m}^{2}$ (Fig. 2).

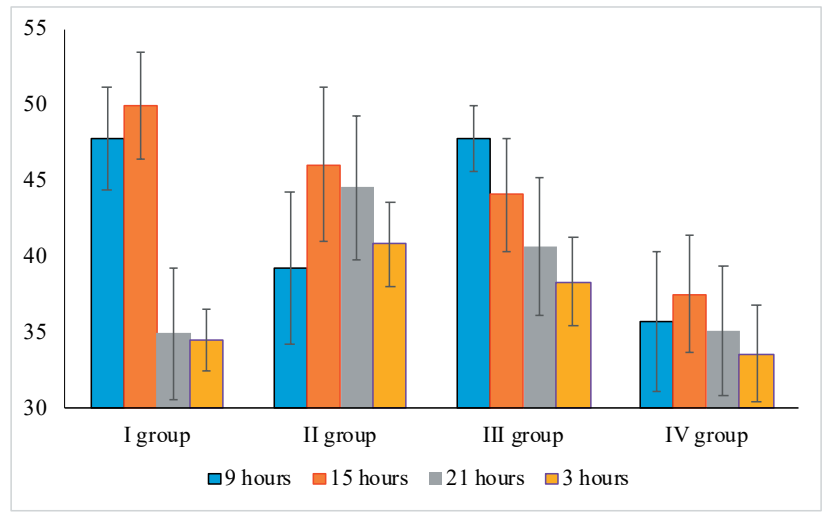

Figure 1. Daily rhythm of the cross-sectional area of hepatocyte nuclei of rats.

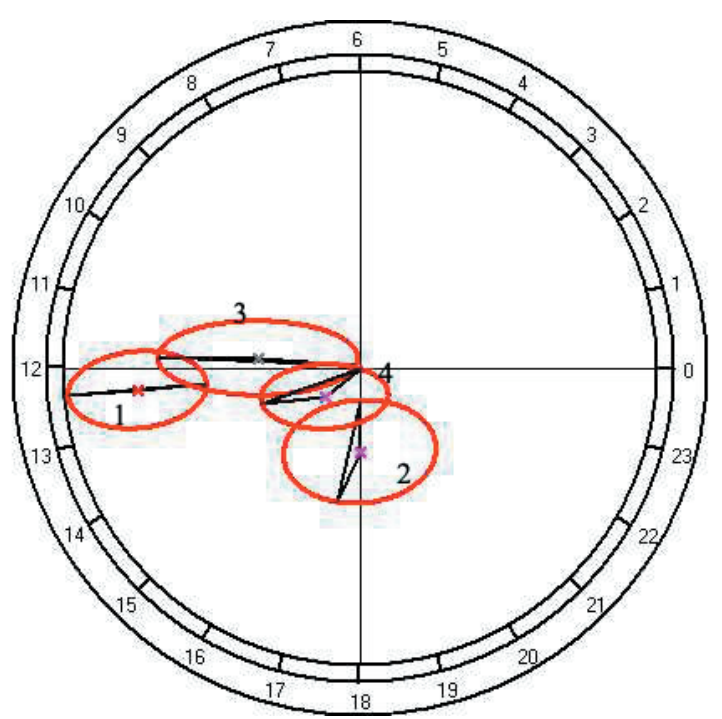

Figure 2. Results of cosinor analysis of circadian rhythmicity of area of nuclei of hepatocytes of rats.

Considering the results of measuring the ploidy of mononuclear hepatocytes, it was found that the studied samples contain diploid, tetraploid and octoploid cells. The average ploidy of the studied hepatocytes is $4.47 \pm 2.12 \mathrm{n}$ in the first group, $5.02 \pm 2.18 \mathrm{n}$ in the second, $4.04 \pm 2.16 \mathrm{n}$ in the third, and $5.18 \pm 2.14 \mathrm{n}$ in the fourth.

Analysis of ploidy distribution of hepatocyte nuclei revealed significant intergroup differences (Table 1).

In particular, in groups in which animals were exposed to chronic alcohol intoxication, the number of diploid hepatocytes significantly decreases, but at the same time, the proportion of octoploid cells in the second group significantly increases, as well as and the proportion of tetraploid cells in the fourth group. 


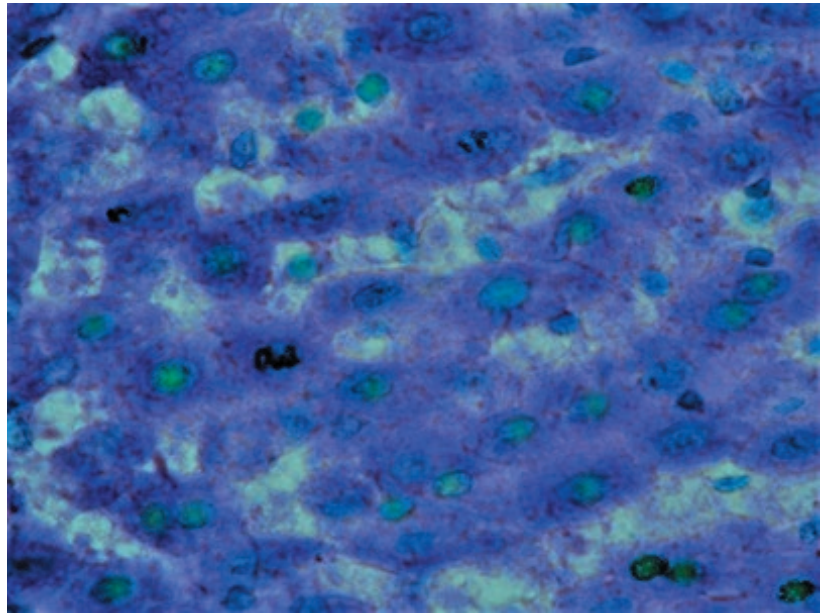

Figure 3. Liver of rat of control group, methylene-green - pyronin $\mathrm{G}, \times 400$.

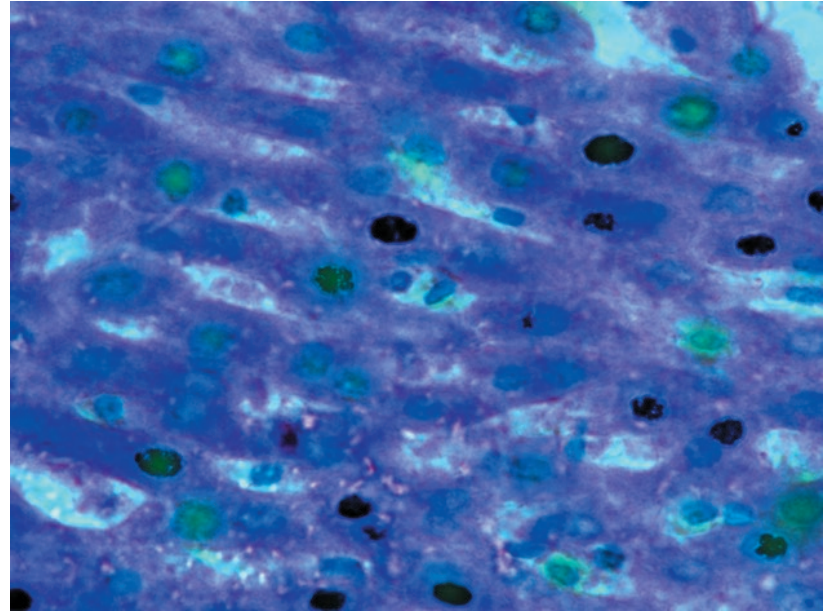

Figure 4. Liver of rat of IV group, methylene-green - pyronin G, $\times 400$.

Table 1. Distribution of hepatocyte nuclei in rat liver depending on ploidy.

\begin{tabular}{|c|c|c|c|}
\hline \multirow{2}{*}{ Group } & \multicolumn{3}{|c|}{ Ploidy of nuclei of hepatocytes } \\
\hline & $2 n, \%$ & $4 n, \%$ & $8 n, \%$ \\
\hline 1st group $(\mathrm{n}=40)$ & $23.98 \pm 3.51$ & $52.1 \pm 4.60$ & $23.23 \pm 2.20$ \\
\hline 2nd group $(n=40)$ & $14.15 \pm 2.02^{* * *}$ & $53.47 \pm 5.18$ & $32.38 \pm 3.21^{* * *}$ \\
\hline 3 rd group $(n=40)$ & $34.0 \pm 4.81^{* * *}$ & $45.9 \pm 3.95^{\star * *}$ & $20.1 \pm 1.89^{\star * *}$ \\
\hline 4 th group $(n=40)$ & $13.70 \pm 2.84^{* * *}$ & $79.6 \pm 5.18^{\star * *}$ & $6.70 \pm 0.81^{\star * \star}$ \\
\hline
\end{tabular}

Note: hereinafter: ${ }^{*}(\mathrm{P} \leq 0.05) ;{ }^{* *}(\mathrm{P} \leq 0.005) ;{ }^{* *}(\mathrm{P} \leq 0.0005)$ - statistical significance of differences in comparison with the control group.

\section{DISCUSSION AND CONCLUSION}

The conducted study allowed us to establish that both the violation of the light regime and the effect of ethanol, individually and jointly, have a significant effect on the studied parameters.

The alcoholic intoxication at fixed light regime causes the decrease in proportion of diploid cells with a simultaneous increase in the proportion of octaploid cells.

Changing of the normal light regime to constant light leads to a change in the nature of the ploidy distribution of hepatocytes. The increase in proportion of diploid hepatocytes indicates a successful course of adaptation processes in the organ, apparently due to the division of cells of higher ploidy, the proportion of which has decreased (Nagy et al., 2001; Yelchaninov et al., 2011; Lazzeri et al., 2019).

The alcoholic intoxication in conditions of constant lighting lead to decrease in size of nuclei and increase in proportion of tetraploid hepatocytes.

The increase in general ploidy in groups 2 and 4 (i.e. in those where animals were exposed to alcohol) occurs due to tetra- and octaploid nuclei, which, according to a number of authors, indicates the development of hepatocyte hypertrophy against the background of an increase in nuclear ploidy (Miyaoka et al., 2012; Zhou et al., 2016). It has been suggested that the polyploid state functions as a growth suppressor, limiting the proliferation of most of cells and causing compensatory-adaptive reactions in the form of diploid cell hypertrophy.

In turn, the nature of the circadian rhythm of the size of the cell nuclei indicates that constant illumination and ethanol, acting separately, cam use a rearrangement of the $\mathrm{CR}$, but the combined action of these parameters leads to the destruction of the circadian rhythm, which indicates a disruption of adaptation processes in animals of this group (Maruani et al., 2018; Matkarimov, 2020)

So, the conducted study testifies that the results of caryometric and ploidometric studies characterize the degree of influence of alcohol intoxication and changes in the light regime on the liver of rats, representing the degree of effectiveness of adaptation to these factors. 


\section{ACKNOWLEDGEMENTS}

Financial support for this study was carried out by Research Institute of Human morphology.

\section{COMPLIANCE WITH ETHICS GUIDELINES}

All the experimental protocols were performed in accordance to ethical guidelines approved by the Research and Ethics Committee of Scientific Center for Biology of Cells and Applied Biotechnology of the Moscow State Regional University, Moscow, Russian Federation prior executing the experiments. Experiments were performed as per "Directive 2010/63/EU of the European Parliament for animal use for scientific purpose" and "NIH Guidelines for the Care and Use of Laboratory Animals".

\section{REFERENCES}

Aizawa S, Brar G, Tsukamoto H. 2020. Cell Death and Liver Disease. Gut Liver. 14(1):20-29.

Beauvalet, J. C., Quiles, C. L., de Oliveira, M. A. B., Ilgenfritz, C. A. V., Hidalgo, M. P. L., \& Tonon, A. C. 2017. Social jetlag in health and behavioral research: a systematic review. ChronoPhysiology and Therapy, 7, 19-31.

Broeke J., Pérez J. M. M., Pascau J. 2015. Image processing with ImageJ. - Packt Publishing Ltd., 259 p.

Clemens MM, McGill MR, Apte U. 2019. Mechanisms and biomarkers of liver regeneration after druginduced liver injury. Adv Pharmacol. 85:241-262.

Cornelissen G. 2014. Cosinor-based rhythmometry. Theor Biol Med Model. 11:16.

Davis BT 4th, Voigt RM, Shaikh M, Forsyth CB, Keshavarzian A. 2018. Circadian Mechanisms in Alcohol Use Disorder and Tissue Injury. Alcohol Clin Exp Res. 42(4):668-677.

DeBruyne JP, Weaver DR, Dallmann R. 2014. The hepatic circadian clock modulates xenobiotic metabolism in mice. J Biol Rhythms. 29(4):277-87.

Ding G, Gong Y, Eckel-Mahan KL, Sun Z. 2018. Central Circadian Clock Regulates Energy Metabolism. Adv Exp Med Biol. 1090:79-103.

Donne R, Saroul-Aïnama M, Cordier P, Celton-Morizur S, Desdouets C. 2020. Polyploidy in liver development, homeostasis and disease. Nat Rev Gastroenterol Hepatol. 17(7):391-405.

Duncan AW. 2013. Aneuploidy, polyploidy and ploidy reversal in the liver. Semin Cell Dev Biol. 24(4):347-56.
El-Sokkary GH, Abdel-Rahman GH, Kamel ES. 2005. Melatonin protects against lead-induced hepatic and renal toxicity in male rats. Toxicology. 213(1-2):25-33.

Esperandim VR, da Silva Ferreira D, Saraiva J, Silva ML, Costa ES, Pereira AC, Bastos JK, de Albuquerque S. 2010. Reduction of parasitism tissue by treatment of mice chronically infected with Trypanosoma cruzi with lignano lactones. Parasitol Res. 107(3):525-30.

Filiano AN, Millender-Swain T, Johnson R Jr, Young ME, Gamble KL, Bailey SM. 2013. Chronic ethanol consumption disrupts the core molecular clock and diurnal rhythms of metabolic genes in the liver without affecting the suprachiasmatic nucleus. PLoS One. 8(8):e71684.

Gilgenkrantz H, Collin de l'Hortet A. 2018. Understanding Liver Regeneration: From Mechanisms to Regenerative Medicine. Am J Pathol. 188(6):1316-1327.

Gillette MU. 2013. Introduction to biological timing in health and disease. Prog Mol Biol Transl Sci. 119:XIXIV.

Huang MC, Ho CW, Chen CH, Liu SC, Chen CC, Leu SJ. 2010. Reduced expression of circadian clock genes in male alcoholic patients. Alcohol Clin Exp Res. 34(11):1899-904.

Junatas KL, Tonar Z, Kubíková T, Liška V, Pálek R, Mik P, Králíčková M, Witter K. 2017. Stereological analysis of size and density of hepatocytes in the porcine liver. J Anat. 230(4):575-588.

Lazzeri E, Angelotti ML, Conte C, Anders HJ, Romagnani P. 2019. Surviving Acute Organ Failure: Cell Polyploidization and Progenitor Proliferation. Trends Mol Med. 25(5):366-381.

Li W, Li L, Hui L. 2020. Cell Plasticity in Liver Regeneration. Trends Cell Biol. 30(4):329-338.

Lunn RM, Blask DE, Coogan AN, Figueiro MG, Gorman MR, Hall JE, Hansen J, Nelson RJ, Panda S, Smolensky MH, Stevens RG, Turek FW, Vermeulen R, Carreón T, Caruso CC, Lawson CC, Thayer KA, Twery MJ, Ewens AD, Garner SC, Schwingl PJ, Boyd WA. 2017. Health consequences of electric lighting practices in the modern world: A report on the National Toxicology Program's workshop on shift work at night, artificial light at night, and circadian disruption. Sci Total Environ. 607-608:1073-1084.

Makovicky P, Tumova E, Volek Z, Arnone JM, Samasca G, Makovicky P. 2018. The relationship between hepatocytes and small bowel after early and short food restriction: What the results show in morphometry. Bratisl Lek Listy. 119(3):156-159.

Matkarimov R. 2020. Questions of temporary adaptation of weightlifters to different climatic and geographical conditions. Euras. J of Sport Sc. 1(1):18-22. 
Martínez-Salvador J, Ruiz-Torner A, Blasco-Serra A, Martínez-Soriano F, Valverde-Navarro AA. 2018. Morphologic variations in the pineal gland of the albino rat after a chronic alcoholisation process. Tissue Cell. 51:24-31.

Maruani J. 2018. The neurobiology of adaptation to seasons: relevance and correlations in bipolar disorders. Chronobiol. Int. 35(10):1335-1353.

McKenna H, van der Horst GTJ, Reiss I, Martin D. 2018. Clinical chronobiology: a timely consideration in critical care medicine. Crit Care. 22(1):124.

Miyaoka Y, Ebato K, Kato H, Arakawa S, Shimizu S, Miyajima A. 2012. Hypertrophy and unconventional cell division of hepatocytes underlie liver regeneration. Curr Biol. 22(13):1166-75.

Nagy P, Teramoto T, Factor VM, Sanchez A, Schnur J, Paku S, Thorgeirsson SS. 2001. Reconstitution of liver mass via cellular hypertrophy in the rat. Hepatology. 33(2):339-45.

Roenneberg T, Merrow M. 2016. The Circadian Clock and Human Health. Curr Biol. 26(10):R432-43.

Roenneberg T, Pilz LK, Zerbini G, Winnebeck EC. 2019. Chronotype and Social Jetlag: A (Self-) Critical Review. Biology (Basel). 8(3):54.

Rosenwasser AM. 2015. Chronobiology of ethanol: animal models. Alcohol. 49(4):311-9.

Stubblefield, J.J., Green, C.B. 2016. Mammalian Circadian Clocks and Metabolism: Navigating Nutritional Challenges in a Rhythmic World. In Circadian Clocks: Role in Health and Disease (pp. 153-174). Springer, New York, NY.

Tahara Y, Aoyama S, Shibata S. 2017. The mammalian circadian clock and its entrainment by stress and exercise. J Physiol Sci. 67(1):1-10.

Tahara Y, Shibata S. 2016. Circadian rhythms of liver physiology and disease: experimental and clinical evidence. Nat Rev Gastroenterol Hepatol. 13(4):21726.

Tong X, Yin L. 2013. Circadian rhythms in liver physiology and liver diseases. Compr Physiol. 3(2):917-40.

Trefts E, Gannon M, Wasserman DH. 2017. The liver. Curr Biol. 27(21):R1147-R1151.

Walker WH 2nd, Walton JC, DeVries AC, Nelson RJ. 2020. Circadian rhythm disruption and mental health. Transl Psychiatry. 10(1):28.

Wasielewski JA, Holloway FA. 2001. Alcohol's interactions with circadian rhythms. A focus on body temperature. Alcohol Res Health. 25(2):94-100.

Yelchaninov AV, Bolshakova GB. 2011. Size of hepatocytes and their nuclei in the regenerating fetal liver of rats // Russian medical and biological bulletin named after academician IP Pavlov. 2(2):128-180.
Zhang S, Lin YH, Tarlow B, Zhu H. 2019. The origins and functions of hepatic polyploidy. Cell Cycle. 18(12):1302-1315.

Zhou D, Wang Y, Chen L, Jia L, Yuan J, Sun M, Zhang W, Wang P, Zuo J, Xu Z, Luan J. 2016. Evolving roles of circadian rhythms in liver homeostasis and pathology. Oncotarget. 7(8):8625-39. 